\title{
Integrating effective learning strategies in basic physics lectures: A thematic analysis
}

\author{
Vegard Gjerde $\odot$, Bodil Holst $\odot$, and Stein Dankert Kolstø॰ \\ Department of Physics and Technology, University of Bergen, 5007 Bergen, Norway
}

(Received 20 November 2020; accepted 25 February 2021; published 14 April 2021)

\begin{abstract}
Introductory mechanics is an obligatory course for many disciplines outside of physics and the failure rate is often high. Even the students who pass the course often fail to achieve the main learning goal: The conceptual knowledge required for modeling situations with physics principles. In many cases, this is due to inefficient learning strategies. However, little work has been done on the integration of learning strategies into physics courses. Here we present a study where we integrated three established learning strategies into the teaching of an introductory mechanics course: elaborative encoding, retrieval practice, and selfexplanation. We also developed three scaffolding tools to facilitate the integration of the strategies: elaborative encoding questions, a hierarchical principle structure, and a problem-solution structure with emphasis on physics modeling. The overarching aim of this study is to use students' experience-based reflections to find ways to improve the integration of the learning strategies. To fulfill this aim, we seek answers to the following three interrelated research questions: (i) What are the students' experiences and associated reflections with the learning strategies and tools? (ii) How do the students' experiences and reflections align with established theory on the learning strategies? (iii) What main barriers to effective implementation of the learning strategies may be hypothesized? To answer these questions, we did semistructured research interviews with 12 students. Through the thematic analysis, we found that retrieval practice was used extensively while self-explanation and elaborative encoding was partly or wholly ignored. The analysis, together with theoretical considerations, indicates that successful implementation of elaborative encoding is critical for maximizing the benefits from retrieval practice in physics. We also present some promising findings on two of the tools: (i) Students' extensive use of the hierarchical principle structure and (ii) that some students started practicing physics modeling after exposure to the solution structure. Finally, we offer suggestions for how to overcome the barriers to effective implementation of each of the learning strategies.
\end{abstract}

DOI: 10.1103/PhysRevPhysEducRes.17.010124

\section{INTRODUCTION}

Learning how to model a situation with physics is arguably the most important thing physics students can learn [1,2]. When students make an effort to model the situation in a problem by using physics principles, they activate and contextualize prior knowledge, deepening their conceptual understanding. For reference, we use physics principles to refer to both the fundamental principles (Newtons' laws and conservation of energy) and derivable principles (e.g., work-energy theorem). However, many physics students use a formula-hunting, plug-and-chug approach to problem solving $[3,4]$. Consequently, many students fail to acquire conceptual knowledge $[5,6]$, even after finishing courses at prestigious universities $[7,8]$ and

Published by the American Physical Society under the terms of the Creative Commons Attribution 4.0 International license. Further distribution of this work must maintain attribution to the author(s) and the published article's title, journal citation, and DOI. even after solving hundreds of physics problems [9]. Many physics students are not even able to reproduce Newton's second law, the work-energy theorem, or other important physics principles a few weeks before the final exam in mechanics [10]. Consequently, the failure rate is often high and the main learning goals are not achieved, even if the students have devoted a considerable amount of study time.

Cognitive science and educational psychology, together with physics education research, provide many insights into the cognitive knowledge structure students need to acquire for physics principles. For example, students need to build useful associative links within and between physics principles [11-13]; they need to build adequate memory strength for physics principles [10,11]; they need to integrate physics principles into a hierarchical cognitive structure [11,13-15]; they need to build the conceptual knowledge required for physics modeling when studying worked examples [16-18]; and they need to learn how to model a situation with physics principles $[1,15,19,20]$. Cognitive science and educational psychology can provide learning strategies that are well suited for stimulating 
learning on all these levels, e.g., Refs. [21,22]. However, there is a lack of translation from basic research to the teaching of physics [23].

There have been numerous attempts at intervening in students' use of learning strategies in higher education. However, students often hold widespread beliefs and illusions about learning that hinders them from becoming self-regulated users of effective learning strategies [24]. They may also struggle to transfer the learnings from oneoff interventions from outside the classroom to inside the classroom [25] and, although they often intend to use effective learning strategies, many fail to follow through with their intentions [26]. Many students tend to prefer ineffective learning strategies-e.g., reading, copying, and highlighting lecture notes [26] — over effective learning strategies [27-29], even when given direct instruction in the relative effectiveness of learning strategies and when given evidence that their performance improved with effective strategies [30]. Yan, Bjork, and Bjork [30] argued that the three main reasons for students' persistent misconceptions of learning were the sense of fluency associated with ineffective strategies, preexisting beliefs, and thoughts about unique learning styles.

There seems to be a growing consensus that training inside the context of the classroom is superior to interventions outside of the classroom $[25,31,32]$. However, few studies on learning strategies are conducted in authentic classroom settings $[33,34]$. Fewer still implement multiple learning strategies. A rare exception being Gurung and Burns [35] who implemented two learning strategiesretrieval practice and distributed practice-in an authentic setting. Biwer et al. [36] used a 6-h intervention, outside of the classroom, to foster students' awareness, reflection, and practice of multiple learning strategies in medical sciences. The qualitative findings suggested that student adoption was impeded by the students' uncertainties about effort and time management, lack of available material, and lack of knowledge of how to implement the learning strategies. Also, outside of the classroom, Endres et al. [37] tried to follow multiple proven principles for strategy interventions [38] in their digital learning environment, such as implementation intentions [39] and principles for multimedia learning [40]. Still, most students continued with suboptimal studying.

In addition to the challenges mentioned above, it is difficult to teach students the relevant and necessary knowledge about the learning strategies. Moreover, it is difficult to bridge the gap from correct knowledge to actual usage of the learning strategies [30], especially when introducing multiple learning strategies [36]. Further, the structure of the domain knowledge is opaque to the students [41], making it difficult for them to find and use suitable educational resources.

Basic learning strategies can potentiate more complex learning strategies. For example, elaborative encoding can potentiate retrieval practice, retrieval practice can potentiate self-explanations, and self-explanations can potentiate problem solving. This is an important reason why simultaneous integration of several strategies is critical for achieving the potential of each strategy. Integration is also important for identifying relevant barriers and facilitators for each learning strategy. See Secs. I A-IC for further discussion on how the learning strategies potentiate each other.

In this study, we integrated three established learning strategies into two existing learning arenas of an introductory mechanics course: Lectures and a problem-solving seminar. We developed three scaffolding tools to facilitate the integration. By integrating learning strategies into the learning arenas of a course, we ensure that more students get exposure and practice over an extended period of time, with the possibility of slowly changing their study habits. Specifically, the three strategies were elaborative encoding for learning useful associative links within and between physics principles; retrieval practice for strengthening the memories of physics principles; and self-explanation for learning the conceptual knowledge required for physics modeling during problem solving. The three associated scaffolding tools were a set of questions for supporting elaborative encoding, the hierarchical principle structure for mechanics [10], and a problem-solution structure with emphasis on physics modeling; see Supplemental Material [42] for more information on the scaffolding tools. Prior work has shown that retrieval practice of the hierarchical principle structure for mechanics is beneficial for the performance of physics students [10]. Our study differs from this and other studies on learning strategies in that we integrate multiple strategies into lectures, all intended to synergize, and that we focus on effective implementation.

This study is not a test of whether these learning strategies work in physics. An important assumption of our work is that the presented learning strategies are indeed effective, as shown by basic cognitive science and educational psychology, e.g., Refs. $[21,22,43]$. The overarching purpose of this study is to investigate how to effectively implement the integration of these strategies and tools into introductory physics courses, especially by identifying important barriers and facilitators.

In order to achieve our purpose, we pursue three interrelated research questions: (i) What are the students' experiences and associated reflections with the learning strategies and tools? (ii) How do the students' experiences and reflections align with established theory on the learning strategies? (iii) What main barriers to effective implementation of the learning strategies may be hypothesized? To answer these questions, we used thematic analysis [44] to qualitatively analyze semistructured research interviews with 12 students. Qualitative analysis is an established tool for investigating the processes of an intervention [45]. 
Thematic analysis is a foundational type of qualitative analysis where one identifies, checks and rechecks, progressively refines, and reports themes in the data [44]. We use the framework of knowing what to do when, why, and how ["WWW\&H," e.g., Refs. [31,46] ]—important aspects of self-regulated learning-for comparing students' metacognitive knowledge of the learning strategies with established theory. The university was shut down because of the Covid-19 pandemic eight weeks into the 22-week semester. This changed the study into a two-month intervention, where they lost access to some of the structured learning arenas and self-regulated use of learning strategies became more important.

In the next sections, we introduce the three learning strategies of elaborative encoding, retrieval practice, and self-explanations. The scaffolding tools are briefly discussed in the methods section.

\section{A. Learning strategy 1: Elaborative encoding}

Elaborative encoding is to deliberately search for connections between knowledge units, to create redundancy of retrieval cues to the memory and redundancy of inference pathways to the memory [47]. Elaborative encoding works best when students create highly integrated, plentiful, meaningful, and predictive associative links [47-49]. This can be done by answering guiding questions that are intended to stimulate elaborative encoding [50]. An example elaborative encoding-question is "What happens if one variable in the principle changes size?"

When students first start learning new physics principles, they lack meaningful associations between the symbols and terms within the equations and they lack meaningful associations between principles [48]. Therefore, students have to rely on cue strength, which they also lack, to be able to retrieve a weak memory [51]. Creating meaningful connections within and between principles gives students more direct retrieval pathways and more ways to reconstruct weak memories during retrieval practice $[12,47]$. Hence, we believe that elaborative encoding of physics principles can potentiate retrieval practice through the creation of intra- and interitem associations because it enables mental reconstruction of the memory $[12,49]$ and increases the retrieval success rate during retrieval practice [52-54]. Self-evidently, meaningful associative links within and between physics principles are also important for achieving mastery of physics modeling.

\section{B. Learning strategy 2: Retrieval practice}

Retrieval practice is a learning strategy where one purposefully retrieves memories in order to strengthen them and increase the likelihood of being able to recall them at a later stage, e.g., being able to recall Newtons' three laws. Retrieving a memory adds more strength to the memory compared to restudy [55] because, unlike restudy, retrieval is a gradual process of reconstructing the memory thereby increasing the strength of intra-item associations $[12,56]$. The increased memory strength from retrieval practice improves memory accessibility [51], makes retrieval of the memory less dependent on cues from the environment [12,51], spares working memory capacity [57-59], improves retrieval fluency [60], and improves transfer to new contexts [34,61-63]. Despite the proven benefits of retrieval practice in many domains [21,22], little research has been done on retrieval practice in physics until recently $[10,17,23]$.

Retrieval practice of physics principles and their conditions of application can potentiate other learning strategies [64-66]. It can reduce the failure rate and time spent floundering during problem solving, especially during physics modeling [67]; it can improve the quality of student problem solving by shifting their focus to the conditions of application of principles [17]; it can improve the quality of students' self-explanations [17] through increased prior knowledge and knowledge access [68-70]; and we speculate that it can also improve the effectiveness of other learning activities, such as reading and attending lectures.

Novice students typically lack cohesion in their knowledge [41]. Some research suggests that a hierarchical structuring of memories enables direct encoding into long-term memory, thereby extending the capacity of working memory through long-term working memory [14]. Gjerde, Holst, and Kolst $\varnothing$ [10] used the meaningful connections between physics principles in mechanics to create a hierarchical principle structure for mechanics, which their students used in retrieval practice. We believe that this is a superior option to using tables, flashcards, or any other structuring of the retrieval material that fails to meaningfully organize the content knowledge.

\section{Learning strategy 3: Self-explanations}

Self-explanation is to explain the steps in a worked example in order to learn how to solve problems. For example, one can explain the physics model in a worked example by identifying the underlying physics principle(s), explicating the conditions of application, and describing how the mathematical equations are set up and why $[16,17,71]$. Self-explanation results in the creation of abstract rules for problem solving that can be retrieved and interpreted during problem solving and in direct memories of parts of worked examples that can be retrieved and used analogically $[16,17,72]$. The abstract rules provide context, direction, and depth to problem-solving actions [17,72], building the conceptual knowledge base required to model physical situations.

High-quality self-explanation-self-explanations that explicate principles, conditions of actions, and goals for the action steps in worked examples-can potentiate problem solving, especially helping students learn how to solve conceptually challenging problems $[16,17,73]$. Self-explanation increases students' reliance on prior 
knowledge [68-70], and may therefore synergize with retrieval practice of physics principles by converting strong memories of physics principles into useful conceptual knowledge [17]. Finally, a major contributor to many students' low learning efficiency during problem solving is time spent floundering [52,74]. Self-explanations can reduce students' floundering through prior self-explained worked examples, but also through treating the problem they are currently stuck on as a worked example (given that the solution is readily available).

\section{METHODS}

\section{A. Participants}

The intervention was implemented in an introductory mechanics course with approximately 150 students enrolled. There were 12 interview participants, seven females, five males, and a mean age of 21 years (range: 19 to 28). There were four students from physics, two from energy, two from geophysics, one from ocean technology, one from nanotechnology, one from teacher education, and one other. There was also a wide range in previous calculus grades ( $\mathrm{F}$ to $\mathrm{B}$ ) and final exam scores in mechanics (19-98 percentage points). The study was approved by the Norwegian Centre for Research Data and all the participants provided informed consent.

\section{B. The learning strategy integration intervention}

We integrated the three learning strategies into lectures and a problem-solving seminar. The existing structure of the lectures was a mix of traditional lecturing and Peer Instruction with conceptual problems [7]. Peer Instruction is a useful way to focus students' attention on the relevant physics principles, as conceptual problems are often designed to reduce the need for mathematics. We kept the existing lecture structure but reduced the traditional lecturing.

We used the first lecture of the semester to briefly inform students about what the learning strategies are, why they improve learning, and how and when they should be used. We also presented some of our results from prior semesters and some important results from the literature. Finally, we told them about the results in the literature of how students tend to prefer lectures and strategies that feel fluent and effortless but which ultimately result in less learning than more effortful and active lectures and strategies $[75,76]$.

\section{Elaborative encoding and retrieval practice integrated into lectures}

The students participated in structured elaborative encoding and retrieval practice during 15-min sessions at the start of one of two weekly lectures. Hence, they could participate in six 15-min sessions in the six weeks leading up to the Covid-19 shutdown. The students retrieved and elaboratively encoded physics principles, using the hierarchical principle structure for mechanics as a scaffolding tool. The students were advised on how to maximize the effectiveness of the retrieval practice through advice on a projector screen and a short instructional video on how to do retrieval practice. The elaborative encoding questions were adjacent to the retrieval practice advice on the projector screen. These questions were intended as a scaffold for creating associative links within and between principles we had not yet covered and for principles they were unfamiliar with. The retrieval practice-advice provided suggestions for when they should spend time answering the elaborative encoding questions. Students performed the retrieval practice on a sheet of paper where all the equations had been fully or partly removed from the hierarchical principle structure. Every student had the full hierarchical principle structure available for feedback and restudy. Students were told that we expected them to do retrieval practice on the material that had already been covered in lectures, but that they could go beyond this if they wanted to. To model a way to answer the elaborative encoding questions, we uploaded example Q\&As for five important principles and notified students of this with an announcement on the student portal. See Supplemental Material [42] for the elaborative encoding-questions, an example Q\&A for elaborative encoding, the hierarchical principle structure for mechanics, the retrieval sheets, and the retrieval practice advice.

\section{Self-explanation integrated into seminars}

Self-explanation was integrated into a weekly 2 -h seminar on problem solving. The students received four weekly seminar problems as voluntary homework one week prior to the seminars. The problems were almost all multipleequation problems and generally not broken into parts (problems from Ref. [77]). The solutions were structured in a way to emphasize the different phases of problem solving, which has been found to facilitate students' understanding and transfer [74,78] and they contained no instructional explanations to avoid suppressing selfexplanation activity [79]. Specifically, we structured the solutions according to the following five steps: (i) Initial coding of the problem by identifying the goal(s) and given variables, (ii) constructing a diagram and/or a picture, (iii) modeling the problem with physics principles, (iv) solving the problem by doing mathematical procedures on the physics model, and (v) reflecting on the solution. See Supplemental Material [42] for an example solution following this structure.

The seminar started with 10 min of individual written self-explanation of a worked example, which the students had not prepared for in advance. This was followed by a short $(5 \mathrm{~min})$ explanation of the worked example by the seminar leader. The students used a worksheet where the whole solution was available, following the solution 
structure above (see Supplemental Material [42] for an example of the solution structure) and were told to write self-explanations in a box under the solution. The four pre-announced seminar problems then followed, which took approximately $15 \mathrm{~min}$ each. For these four problems, the seminar leader first coded the problem and drew a diagram while explaining his thinking. Then, a physics model for the problem (the key equations) was presented on a screen and the students attempted to explain the model. Afterward, the seminar leader presented his explanation of the physics model, i.e., explicating the principles and conditions of application, describing the set up, and explaining how the model could lead to the goal, before revealing and explaining the rest of the solution, i.e., the mathematical procedures and final reflections. In the final $15 \mathrm{~min}$ of the seminar, the students attempted to solve a new problem by using the presented solution structure. The seminar leader progressively revealed and explained parts of his solution to help the students who were stuck. Hence, the students' five opportunities to practice self-explanation and one opportunity to practice physics modeling were interleaved with the seminar leader modeling how to self-explain the physics model six times. By receiving a thorough explanation of the physics model, the students were also indirectly taught how to do physics modeling.

After the shutdown, the problem-solving seminar was uploaded as on-demand videos, with one to two videos per problem. The structure of the seminar was the same. However, instead of the allotted time for students' selfexplaining in the physical seminars, the students were rather encouraged to pause the video and self-explain in the digital seminars. Seven out of the fourteen problem-solving seminars were digital.

The solution sheet to the six problems was uploaded immediately after the seminar. Although not all students were aware of this, the students could access the textbook solution manual for the four pre-announced seminar problems (with a more standard solution structure) to help them prepare for the seminars. This was not possible for the first and the last problem during the seminar, as these were not pre-announced and were frequently adapted from a different textbook or prior exam questions.

\section{Interview procedures}

The semistructured research interviews were conducted digitally with an interview guide by the third author. This was to reduce potential bias due to students' familiarity with the first author (problem-solving seminar leader) and the second author (the lecturer). The interview guide consisted of questions that probed students' experiences and reflections of the different learning strategies and tools, see Supplemental Material [42] for the interview guide. Intelligent verbatim transcription of the interviews was done by an authorized company (Totaltekst).

\section{Data analysis}

We used a variant of the thematic analysis method laid out by Braun and Clarke [44]. The analysis was performed in the software NVivo [80]. Our thematic analysis identified themes explicating and naming meanings expressed by the students, with some themes representing our interpretation of the underlying meaning of the students' utterances. We counted as a theme anything that captured important aspects related to our research questions, especially looking for themes important for improving the integration of the learning strategies and tools. We use the framework of knowing what to do when, why, and how, e.g., Refs. [31,46] for analyzing the alignment between students' metacognitive knowledge of the learning strategies and established theory. Similar to Biwer et al. [36], we identify potential barriers and facilitating aspects for each of the learning strategies based on the identified themes.

An important theoretical assumption for the analysis is that people are more similar than dissimilar in their learning processes and that supposed learning styles are irrelevant for optimal instruction and learning (prior knowledge is relevant) [81]. Further, we are not probing for students' unique insights into learning, rather sticking closely to theoretical models of learning and comparing students' practices and reflections to these ideals.

The general flow of the analysis was as follows: The first author listened to all the audio recordings twice before segmenting the written transcripts into broad categories relating to the different learning strategies and tools. The next step was detailed coding of the students' utterances and identification of themes. These codes were then hierarchically categorized according to tentative themes and then structured visually in a thematic map for each learning strategy. The themes were continually refined during the process - changed, collapsed, or separatedin response to renewed inspections of underlying utterances and themes and any perceived lack in internal coherence or external distinguishability, as recommended by Braun and Clarke [44]. The analysis was also discussed in seminars with the first and last author and three other educational researchers. Finally, the sections with qualitative findings and discussion of the findings were sketched and discussed for possible inconsistencies. The first author listened five times through all the interviews during the process, searching for missed themes and inconsistencies.

We caution that sample-to-population generalization is generally not appropriate for qualitative analysis [82]. Nevertheless, we believe that the findings describe existing experiences and reflections. Furthermore, findings may be used as working hypotheses [83] in new contexts. However, the potential for case-to-case generalization needs to be carefully evaluated by the reader through an analysis of whether the readers' relevant context sufficiently matches the particulars of this study [84]. Finally, analytic generalization, where one relates particular findings from a 
qualitative study to a broader theory, is not dependent on samples and populations $[82,85]$. Therefore, we view this study as providing nuance to theories on how to facilitate students' use of learning strategies.

\section{FINDINGS AND DISCUSSION}

In this section, we present the themes regarding students' experiences and reflections with each of the learning strategies (research question 1), connecting the students' experiences and reflections to theory on what to do when, why, and how, e.g., Refs. [31,46] and to barriers and facilitating aspects of the implementation (research questions 2 and 3). We also offer suggestions for how to overcome the barriers and build on facilitating aspects of effective implementation of each of the learning strategies. After presenting the findings from the three learning strategies, we present some emergent findings for the solution structure and physics modeling and for the hierarchical principle structure for mechanics (HPSM). See Supplemental Material [42] for the original quotes in Norwegian.

\section{A. Elaborative encoding}

The identified themes for the students' experiences and reflections with elaborative encoding are listed in Table I, together with their connections to research questions 2 and 3.

\section{Themes in students' experiences and reflections with elaborative encoding}

Three students had vague notions that elaborative encoding was something else, e.g., explaining solutions to oneself or discussion prompts for problem solving, and two students believed that elaborative encoding was best for repetition and testing oneself. Five students thought the cost of doing elaborative encoding was too high and that the value was too low, e.g., "It is so much for so little, I think" and "I guess it is because I haven't felt a need for it. And maybe that I couldn't bother spending time on it, in a way."

Almost without exceptions, the students ignored the elaborative encoding questions and were completely unaware of the example Q\&As we had uploaded.
However, it appeared that about half the students unintentionally practiced elaborative encoding in their self-study, seemingly without awareness that they were doing what was intended with the elaborative encoding questions. One student, who tried to say what he did instead of elaborative encoding, explained exactly what the authors intended for the elaborative encoding:

I think it comes more out of the principle. [...] I think I rather use the physical symbols in the formula, or principle, to remember what it really says and how it looks. Instead of going back and looking [at the elaborative encoding-questions], 'Ok, this equation gives joule, this is then this and this and this.' I think it is more like that.

Two students cited the overwhelming amount of study options as a reason for not engaging in elaborative encoding.

\section{Alignment with theory}

Themes 1-3 in Table I indicate that students gained very little metacognitive knowledge of elaborative encoding. They lacked knowledge of when and why one should use elaborative encoding and many even lacked knowledge of what it is. Theme 4 also indicates that some didn't know how to do it. The pervasive lack of metacognitive knowledge suggests that they also failed to connect elaborative encoding to retrieval practice and that this may have exasperated the problem of rote rehearsal during retrieval practice (see the next section) which is known to be an ineffective strategy [86].

Although it appears that the students lacked metacognitive knowledge of elaborative encoding, it also seems that some students have study practices that align with theory on how one should do elaborative encoding.

\section{Barriers and facilitating aspects}

Theme 3 in Table I indicates that we first need to give students clear reasons for engaging in elaborative encoding. Themes 4-7 indicate that there are substantial barriers to overcome regarding the task structure and associated instructional resources. The students' reluctance to using

TABLE I. Themes from students' experiences and reflections on elaborative encoding (EE).

Themes in students' experiences and reflections

1. Thinks EE is something else than it is

2. Better for repetition and testing

3. Too little value, too high cost

4. Ignored EE-questions

5. Unaware of uploaded example Q\&As

6. Unintentionally using EE during self-study

7. Overwhelmed by study options
Connection to research questions 2 and 3

Lacking knowledge of what it is

Lacking knowledge of when one should use it Lacking knowledge of why one should use it Barriers regarding task structure and resources Barriers regarding task structure and resources Barriers regarding task structure and resources Barriers regarding task structure and resources 
the questions signals a need for improvement in either the task structure (elaborative encoding during structure retrieval practice), the instructional resource (the questions), or both. Theme 5, 6, and 7 indicate that there is a substantial barrier to overcome regarding students' need to self-regulate in an environment with many study options. If we do not provide more structure, we fear that only strong students will study effectively. We failed to identify specific facilitating aspects for elaborative encoding.

\section{Discussion on future practice}

We believe that for elaborative encoding to become effective, we need to develop instructional material that makes it very clear why and how to elaboratively encode physics principles. Based on our findings, it seems unrealistic to get a large proportion of the students to engage in elaborative encoding merely by using direct instruction in metacognitive knowledge and without using structured tasks. Moreover, we need to make this process less effortful and more engaging, preferably removing the need for selfregulation as some students are overwhelmed by the number of study options.

We speculate that it would be fruitful to embed elaborative encoding in a mandatory social learning task [87]. For example, by getting the students to do structured elaborative encoding in randomly generated digital discussion groups, e.g., with three to five students discussing translations between multiple representations of physics principles (graphs, diagrams, equations, etc.) on Zoom or a similar digital platform, and with mandatory uploading of the discussion. We further speculate that this could stimulate more elaborative encoding during self-study, making it more reflective, because the students become more aware of possible intra- and interitem links. Indeed, one student reported being cognizant of the elaborative encoding questions while reading the textbook.

We also speculate that elaborative encoding is best implemented as a separate activity prior to retrieval practice [88], both because many students complain about a lack of understanding during retrieval practice and because we want to ensure memory reconstruction during retrieval practice. Students may then learn how to elaboratively encode principles and start using it for learning new principles during structured retrieval practice. Although some students unintentionally practice elaborative encoding in their self-study, we do not know how many do it, when they do it, or how they do it.

\section{B. Retrieval practice}

The identified themes for the students' experiences and reflections with retrieval practice are listed in Table II, together with their connections to research questions 2 and 3.

\section{Themes in students' experiences and reflections with retrieval practice}

About half the students found retrieval practice of the hierarchical principle structure beneficial in various ways. Encouragingly, some students became aware of more subtle benefits like how the increased familiarity helped them understand more complex material and that they had increased (cognitive) accessibility to the principles. One student said

Say you know these equations already now, and then you come to a lecture, you've seen the equation before, you don't become like *gasping sound* when you see the equation, but 'oh yeah, that is that and this. Cool, yeah, that is this law.' And then they explain why it's like that, and then just 'oh, yeah. Cool.'

They also reported that the benefits were gradually increasing. Four students reported having continued doing retrieval practice after the Covid-19 shutdown, citing as reasons that they wanted to refresh and maintain knowledge and that it was an effective way to kickstart self-study when willpower was lacking. One student said,

When you sit at home in a little room all day you can struggle with your concentration. It is hard to start because you know you have to sit for a long time. So, I

TABLE II. Themes from students' experiences and reflections on retrieval practice (RP).

Themes in students' experiences and reflections

Connection to research questions 2 and 3

Positive themes

1. Finds RP increasingly beneficial

2. Finds RP enjoyable and motivating

3. Elaborating connections helps RP

4. Followed RP advice

Negative themes

5. Ignored RP advice

6. Lack of understanding makes RP rote and unhelpful

7. Limited or low value of RP
Gained knowledge through practice of why one should do RP Facilitating aspect

Facilitating aspect

Facilitating aspect

Barriers regarding task structure and resources Barriers regarding task structure and resources Lacking knowledge of why one should do it 
have often used it [retrieval practice] as a 'this only takes 15 minutes, so you start with that' and then I often notice that it is easier to start things. Then I can already put a checkmark that I have done something that day.

Two students also reported continuing to retrieve memories during problem solving, which is exactly what we intended.

Four students found retrieval practice enjoyable and motivating. They challenged themselves on speed or amount retrieved in a session, finding the challenge-level appropriate, enjoying the obvious progress. When asked about what is most important when memorizing, one student said,

I am actually bad at it [memorization]. I have never done it [before] because I think it is really boring. Is it incentive you call it? It is fun to fill out a sheet of paper, week after week, to see if you know it. I try to use it as motivation-this week I filled out this much, next week I will fill out more. Then it becomes full eventually.

Three students found that elaborating on connections helps retrieval practice. One student said,

Now that I had to think about things and try to fill them out without looking, it became more like if I wanted to find this, what would be logical to include in the equation? Yes, it has to be acceleration over time. Now I know better how to use it.

Five students reported following the retrieval practice advice and these students were almost invariably the same students that contributed to the positive themes above. Seven students reported ignoring the retrieval practice advice and these students were almost invariably the same students that contributed to the negative themes below.

Six students complained that their lack of understanding of the principles made retrieval practice unhelpful and that it forced them to do rote rehearsal, i.e., repeating the words or symbols without using meaningful connections. One student said, "Then it was just memorization of it. So just looking at it without any sense of purpose or meaning, just try to remember it from session to session."

Four students thought that retrieval practice had limited or low value for learning. Two students thought it was outright ineffective, while two students thought the benefits were short-lived, with one student saying "It is a work you have to continue. If not, you lose the value of it pretty fast, unfortunately."

\section{Alignment with theory}

Five students seemed to have gained knowledge that accords well with the theory of why one should do retrieval practice. Interestingly, four out of the five students who noticed the intended benefits of retrieval practice were also students who engaged in physics modeling during problem solving and three of these students also contributed to the themes of unintentionally using elaborative encoding during self-study and that elaborating on connections helps retrieval practice, the latter being an indication of knowledge of how to do retrieval practice. Although we have to be careful about generalizing quantitative effects with so few students, this clustering of themes accords well with our theoretical expectations that elaborative encoding creates intra- and interitem associative links, which again stimulates memory reconstruction and potentiates retrieval practice, which again helps with physics modeling. These students were also largely the same students that followed the retrieval practice-advice.

\section{Barriers and facilitating aspects}

The most important barrier seems to be that students feel their lack of understanding of the symbols in the principles forces them into rote rehearsal during retrieval practice and that this is unhelpful. The same students ignored the retrieval practice advice and some of them indicated that retrieval practice had low value. Taking into account that these students did not contribute to the themes of unintentionally using elaborative encoding during self-study and elaborating connections helps retrieval practice and that everyone ignored the elaborative encoding-questions, the root cause of these problems seems to be that these students lack meaningful associative links within and between principles. They apparently failed to connect elaborative encoding to retrieval practice and they failed to understand how retrieval practice may help them understand the principles. Despite their apparent misunderstandings, this is something we need to address to realize the full potential of these learning strategies.

\section{Discussion of future practice}

The themes of finding retrieval practice increasingly beneficial and finding it enjoyable and motivating indicate that an important way to facilitate retrieval practice is to ensure that students get sufficient practice with the strategy. It is especially encouraging that some students noticed subtle benefits of retrieval practice. However, it is probably hard to ensure that most students use retrieval practice without using highly structured tasks such as what we used in the first few weeks before the Covid-19 shutdown, incorporating retrieval practice as part of the lectures. Therefore, we suggest that structured retrieval practice during lectures is a way to facilitate students' widespread adoption.

The best way to overcome the barriers to effective use of retrieval practice is probably to ensure quality elaborative encoding by all the students. When quality elaborative encoding has been ensured, we must also ensure that everyone gets adequate practice with retrieval practice. A forceful way to ensure this is to implement a mandatory retrieval test. This test can be performed on the same 
TABLE III. Themes from students' experiences and reflections on self-explanation (SE).

Themes in students' experiences and reflections

1. Unaware that $\mathrm{SE}$ is a learning strategy

2. Low perceived value of SE

3. Choose to do other things when stuck

4. Did not understand the method

5. Prefer an intuitive approach to studying solutions

6. Self-explanation is very costly

7. Likes discussing solutions in social situations
Connection to research questions 2 and 3 retrieval sheet as the retrieval practice towards the end of the semester, where students have to reach a certain criterion, e.g., $70 \%$ correct, to be allowed to take the final exam. This is well within reach for all students and provides them with incentives and clear goals. Ideally, we would want students' practice to be intrinsically motivated. However, we believe this intrinsic motivation will come when they see progress and notice the benefits, in line with our thematic analysis and the results of Abel and Bauml [89].

\section{Self-explanation}

The identified themes for the students' experiences and reflections with self-explanation are listed in Table III, together with their connections to research questions 2 and 3 .

\section{Themes in students' experiences and reflections with self-explanation}

Three students seemed unaware that self-explaining solutions could be considered a learning strategy. When asked about whether she ever tried to explain a solution to a problem to herself, one student said, "No, I do not. I have actually never done that, so I do not really know if it would work."

Four students expressed doubt in the value of selfexplanation, specifically that they perceived low benefit and need for self-explanation, particularly for problem solutions they found easy to understand.

A reoccurring theme was that students chose to do other things than self-explaining when stuck in problem solving. Most students said they would rather try to solve the problem and, when stuck, rather use the solution to find minor hints to help them continue with problem solving. Three students chose to read the textbook or watch digital lectures when stuck. When asked about what she did when stuck on a problem, one student said, "I try to read more, until my face turns green." Four students reported that they could not be bothered with self-explaining solutions. Finally, two students said that studying solutions was their very last resort when stuck. One student said, "It [studying solutions] is the very last resort" and another said, "The solution is often available. But I use it as a 'last resource,' you could say." The one clear exception was a student who said he used self-explanations because it was essential to transfer the knowledge to other problems.

Five students expressed difficulty with understanding the method for self-explanations. One student said about the written self-explanation problems from the seminars:

Many failed to understand this sheet. We sat and wrote those self-explanations during the seminars. And then there was this thing that we should not use numbers, and then there were some who only wrote the procedures, some who only tried to write in words what happened. Yes. It was a bit... I think it was a bit unclear.

Seemingly related to the theme of not understanding the method, six students preferred a more intuitive approach to studying solutions. They talked about how they "tried to understand the steps" and "thought through the solution." Three students also felt that the taught method for selfexplanations was too structured and detailed.

Seven students found self-explanation very costly in terms of the effort and time required, saying, e.g., "I remember that it is not the easiest thing to explain with words what goes on step by step, it is much easier to actually describe what you do with math," "It feels effortful to explain a solution to a problem you have not solved yourself," and "Especially now before the exam I think it takes too much time, really."

Finally, six students said they liked to discuss solutions in social situations. Three students said they liked to discuss solutions in peer groups and three other students said they liked the structured self-explanation practice in the seminars.

\section{Alignment with theory}

The students apparently have a pervasive lack of metacognitive knowledge of self-explanations, with one exception being the student who said that self-explanation is essential for transfer. When they are unable to solve problems, several students use something similar to what Renkl [71] calls anticipative reasoning, which is to look for some hint in the solution to help them solve the problem on their own. This is a good strategy, especially for skill acquisition, but it is probably less effective than selfexplanation for learning the conceptual knowledge needed 
for physics modeling. One student said that he searched for similar examples and tried to analogically map the solution, which is also a reasonably good strategy for skill acquisition [90]. The most concerning finding was that several students chose to read the textbook or watch video lectures when they were stuck on problems, both very inefficient strategies for learning to solve problems due to the low specificity [11,91]. This shows a lack of knowledge of when they should self-explain, namely, when they are unable to solve the problem with current knowledge.

\section{Barriers and facilitating aspects}

A large barrier to overcome is students' lack of knowledge of what self-explanation is, and when, why, and how to do it. There seems to be a pervasive view that it is "wrong" or ineffective to study the solutions to problems. This view is implicit in many students' choices and more explicit in that some students view it as a last resort. Several students said they preferred solving problems to explaining solutions, which is good if they have adequate prior knowledge and skill. However, the two are not mutually exclusive and there is a lot of research on how selfexplanation is more effective than problem-solving in the early phases of learning to solve problems $[92,93]$.

Another major barrier is that students find self-explanation very costly in terms of time and effort. This may also be a reason why they prefer using a more intuitive approach to self-explaining solutions.

A potential facilitating aspect was that several students expressed that they liked to discuss solutions to problems with peers.

\section{Discussion of future practice}

The easiest way to make students aware of self-explanations, and to impart knowledge of when, why, and how to use it, is to hand out a short pamphlet on what selfexplanation is, and when, why, and how to do it. Although this method only helps the interested students, it can reduce the time needed for instruction on metacognitive knowledge during lectures and reduce antagonism due to time spent on extracurricular instruction in metacognitive knowledge of learning strategies. The low perceived value of self-explanations seemed tied to low problem difficulty. Hence, the perceived value of self-explanations may increase for more complex problems.
To reduce the cost associated with self-explanations during self-study, we suggest embedding self-explanation in social learning activities, e.g., by using peer instruction of physics models for complex problems. This requires well-thought-out conceptual problems that stimulate highquality explanation activity. One student said about the peer instruction that "I feel that I learn something, but at the same time I don't feel like I have to spend much effort. I feel that I learn without working." Embedding selfexplanation in social learning tasks, especially if mandatory, can potentially remove the need for self-regulation, bypassing students' implicit beliefs about studying solutions. It might also be a more natural way to learn and internalize how to self-explain. Moreover, it may provide students with social support and both peer feedback and instructor feedback on their explanation quality. To reduce the required effort associated with self-explaining, we may also need to improve the students' early acquisition of domain knowledge. We believe that this is best done by ensuring a highquality implementation of elaborative encoding and retrieval practice and by ensuring high participation in both.

\section{The solution structure and physics modeling}

Getting students to adopt the practice of physics modeling is an overarching goal of our research activities. Therefore, we analyzed students' experiences and reflections regarding the solution structure tool and physics modeling. We identified several facilitators and one main barrier to students' use of physics modeling during problem solving; see Table IV. We do not discuss alignment with theory, as we did not have specific research questions for the solution structure and physics modeling.

\section{Themes in students' experiences and reflections with the solution structure and physics modeling}

It seems that most students had pre-existing problemsolving habits that included some form of coding and drawing a diagram. However, we noticed that some students had adopted our terminology relating to coding, specifically "coding," "goal variables," and "given variables."

Most students mentioned how physics modeling and the focus on principles' conditions of application were new to them, while the rest of the problem-solution structure was similar to what they had learned in high school or what they

TABLE IV. Themes from students' experiences and reflections on the solutions structure and physics modeling.

\begin{tabular}{lc}
\hline \hline Themes in students' experiences and reflections & Connection to research goal \\
\hline 1. Coding and drawing diagrams are prior habits & Neutral \\
2. New awareness and practice through exposure to physics modeling & Facilitating aspect \\
3. Gradually persuaded by the benefits of physics modeling & Facilitating aspect \\
4. Use HPSM as a tool for physics modeling & Facilitating aspect \\
5. Just trying to solve problems & Barrier \\
6. Only reflect when problems are difficult or surprising & Neutral \\
\hline \hline
\end{tabular}


already did. When asked whether his use of the structure was old habits from high school, one student said

We were told [at high school] to set up what we have, what we wanted to find, and then draw a diagram. Then you have sorted the data from the text. That helps a lot. It is the [physics] model that is 'new.'

The same students also reported that they went to the seminars mainly to learn how to structure solutions and that they tried to follow the solution structure in their own problem solving. Two students also said that they had started purposefully looking for the conditions of application for new principles they encountered. Five students reported gradually adopting physics modeling after seeing its benefits. Two students also contrasted these benefits with the problems they experienced when just trying to solve the problems. Three students apparently used the hierarchical principle structure for mechanics as a tool for finding physics principles during physics modeling.

The main negative finding from our analysis was that some students were just trying to solve problems. These students had high-school habits that they were happy with, seeing no reason to learn a new way to solve problems; they viewed physics principles as "formulas"; they were satisfied with getting the correct answer and saw no reason for reflecting on the solution; and they believed physics modeling was something you did on exams. Finally, students only reflect on the solutions when the problem was perceived as difficult or surprising and some students report never reflecting on a solved problem.

\section{Barriers and facilitating aspects for physics modeling}

The main barrier to physics modeling seems to be the poor problem-solving habit of just trying to solve problems. We believe that what worked for the students who adopted physics modeling-exposure and increased awareness of physics modeling and its benefits-will also work for students who just try to solve problems. The solvers may be late adopters who will eventually be persuaded by a critical mass of modelers.

It seems that exposure to the idea of physics modeling through the seminar and seminar problems was enough to persuade a large proportion of the students to adopt the practice. Most students tried to follow the structure or said they already did most of it, while about half the students had also adopted the practice of physics modeling. Physics modeling — with its focus on physics principles and their conditions of application-seems to be a new feature of problem solving for the students, as evidenced by several students mentioning how it was a new feature and no one mentioning already doing it. It also seems to be relatively simple to get an idea of how to do physics modeling, as it seems to be enough to have someone model the process or even to merely see it clearly separated and named in problem solutions. The hierarchical principle structure for mechanics also seems to be a useful tool when trying to model problems. Our findings also indicate that when students try the practice of physics modeling, they notice the benefits and gradually adopt it in favor of just trying to solve problems. We find these results encouraging as it seems relatively easy to get students to consciously adopt physics modeling in their problem solving.

\section{Discussion of future practice}

Our first suggestion is to clearly separate the structural elements of a large proportion of the solutions students are exposed to, especially separating the physics modeling from the mathematical procedures. It is a relatively easy practice to implement, for example, by uploading solutions to weekly problems, and it has a high potential gain. Our experience tells us that the solution structure is more convincing for complex problems that require at least two equations to be solved. Too simple problems and problems that are broken into parts can remove the need for physics modeling [94], rather stimulating formula hunting. Therefore, the most beneficial problems are those that involve multiple equations, especially synthesis problems that span multiple principles $[6,95]$. Indeed, Antonenko et al. [19] found that more complex problems, requiring multiple principles for their solution, were effective for gradually changing some students' habits towards a more physics modeling-based approach.

Our second suggestion is to give the same type of solutions to seminar and workshop leaders and to instruct them in the practice and rationale of physics modeling.

\section{E. The hierarchical principle structure for mechanics}

The hierarchical principles structure for mechanics is a central tool in the integration of the three learning strategies, as it is useful for every learning strategy. Therefore, we did a separate explorative analysis of the students'

TABLE V. Themes from students' experiences and reflections on the hierarchical principle structure for mechanics.

\begin{tabular}{ll}
\hline \hline $\begin{array}{l}\text { Themes in students' experiences } \\
\text { and reflections }\end{array}$ & $\begin{array}{c}\text { Evidence of seamless } \\
\text { integration }\end{array}$ \\
\hline $\begin{array}{l}\text { 1. Use HPSM for problem-solving } \\
\text { 2. Use HPSM during lectures } \\
\text { and reading }\end{array}$ & $\begin{array}{l}\text { Positive } \\
\text { Positive }\end{array}$ \\
$\begin{array}{l}\text { 3. Use HPSM for checking units } \\
\text { and concepts }\end{array}$ & Positive \\
$\begin{array}{l}\text { 4. HPSM is always nearby } \\
\text { 5. High perceived usefulness }\end{array}$ & $\begin{array}{l}\text { Positive } \\
\text { 6. Want minor modifications in HPSM }\end{array}$ \\
7. Use HPSM sparingly & $\begin{array}{l}\text { Implicitly positive } \\
\text { Neutral }\end{array}$ \\
\hline \hline
\end{tabular}


experiences and reflections; see Table V. The conclusion is that the hierarchical principle structure seamlessly integrated with students' study habits, both old and new. We do not discuss alignment with theory or barriers and facilitators, as there is no established theory, there were no major barriers, and there seems to be no need for facilitators.

\section{Themes in students' experiences and reflections with the hierarchical principle structure for mechanics}

Most students reported using the hierarchical principle structure for mechanics during problem solving, which they did in three different ways: They remembered equations from HPSM due to prior use and retrieval practice; they looked up the equations when they failed to remember; and they searched for equations when they were stuck on a problem. We know from the findings in Sec. III D that some students are just trying to solve the problems while other students were trying to do physics modeling. The same students tended to call HPSM the "formula sheet" and the "principle structure," respectively.

Five students reported that they used HPSM during lectures and reading. They used it during peer instruction to check their intuition and to support their arguments. They used the backside of HPSM to check the units and what concepts the symbols were for. Finally, several students reported always having HPSM nearby. Two students actually showed the interviewer where they had hung it up on the wall.

The students' high perceived usefulness for HPSM was a strong piece of evidence for seamless integration. Two students reported that it made it easier to see the deep structure in the course and four students said it gave them a better overview of the course. One student said,

It is with me always. Because it's so nice. I think it's very well structured, like: $\mathrm{Ok}$, this, this, and then you see that 'Ok, a lot of the things are repeated.' For you can see things in parallel: $\mathrm{Ok}$, but this is the same' and then... Yes, it's just ingenious. It's very nice to see that: 'This is what we are going to learn.'

Finally, more than half of the students spontaneously praised the HPSM, saying things like "It is the most beautiful sheet of paper in the world," "I think everyone loves it," and "It has been very useful, very useful."

We interpret the students' requests for minor modifications of HPSM as implicit evidence that HPSM was well integrated into their study. Two students said they wanted some things on the HPSM that were not there and two students did not like that some symbols and subscripts were different from what they were used to.

Three of the students reported not using HPSM that much, with two of them citing as a reason that they did their own compilation of "formulas." However, both of these students switched to HPSM when their notes became too voluminous, with one of them saying:

But I think it is very nice now, when I am starting to know the course, to recognize them and just throw a glance at the sheet and find the formulas I need instead of having to flip through seven different documents on the PC to find just the formula I needed.

The third student said that he used the book during problem solving, but he also reported stopping with problem solving early in the semester because he planned to retake the exam over the summer. Further, he said, "But the sheet is definitely useful, so its use should be continued."

Lastly, one student reported one of the main benefits we were hoping for with the hierarchical principle structure for mechanics:

...after you have used it that many times, that sheet, it makes... The formula sheet, it is somehow saved in your head. So, in the end, I didn't need to use it, because I knew them all.

\section{Discussion of future practice}

We suggest that instructors in mechanics provide students with the hierarchical principle structure for mechanics [10] or a similar hierarchical principle structure. It seems that the hierarchical principle structure for mechanics seamlessly integrates with the students' study habits and that almost everyone chooses to use it. They use it for problem solving, they use it for other study strategies, and it helps them get an overview and to see structural similarities between the different parts of the course. The second author integrated the hierarchical principle structure into her lectures, gradually revealing parts of it as she introduced new principles. A lecturer can also advise students to use it to construct arguments during peer instruction or to use it as a starting point for discussion. In conclusion, the hierarchical principle structure for mechanics is a useful tool that can be adapted for use in any learning activity where physics principles are the core ideas.

\section{GENERAL DISCUSSION AND CONCLUSIONS}

\section{A. Do the findings agree with prior findings?}

The results agree with the literature in that it is difficult to get students to adopt learning strategies and that students tend to have poor metacognitive knowledge of learning strategies. We identified some of the same themes as Biwer et al. [36], namely, students' uncertainties about effort and time management and the lack of knowledge of how to implement the learning strategies. Our results also agree 
with others in that students lack the required self-regulation and that they need support [96]. Our findings generally support the findings of Yan, Bjork, and Bjork [30], in that the students' sense of effort with the learning strategies, preexisting beliefs about learning, and thoughts about learning styles were barriers to their use of effective learning strategies. Renkl et al. [97] discussed three potential reasons why students chose not to use selfexplanations after their short-term training intervention: low perceived usefulness, low saliency of strategy during self-study, and too short intervention. Our findings also indicate low perceived usefulness as a barrier. However, we feel that the main barrier is the high cost of engaging in selfexplanations, with the second-largest barrier being the faulty beliefs about learning implicit in their choosing to do other things.

\section{B. Implications for further integration of learning strategies}

The encoding strategies-elaborative encoding and selfexplanation-appear to be especially effortful and difficult to understand. The task structure of both these strategies seems to be opaque to the students. In Secs. III A and III C, we suggested that these strategies should be embedded in social learning tasks, perhaps mandatory in the case of elaborative encoding. Social learning processes can remove the mental effort barrier and capitalize on additional scaffolds such as providing students with different roles, immediate feedback, and peer modeling. However, both elaborative encoding and self-explanation require further resource and task structure development for effective integration into physics courses.

We found that retrieval practice is easy to implement and that several students kept doing it after the Covid-19 shutdown. However, we highly doubt that many students would adopt retrieval practice if it was merely encouraged and the required resources made available, even if given direct instruction in the what, when, why, and how. Therefore, we believe that the practice of structured retrieval practice in lectures, or something similar, is crucial for ensuring students' use of this strategy.

Most students adopted the problem-solution structure and some students also adopted the practice of physics modeling. We suspect that more students would adopt physics modeling with well-implemented peer instruction using self-explanation of physics models and with a more holistic integration where the provided problem solutions, the lecturer, and the teaching assistants all focus on physics modeling as an essential part of physics. The hierarchical principle structure for mechanics is a useful tool for teaching, learning, and doing physics modeling. It can also be useful for elaborative encoding, retrieval practice, and self-explanation, and for many other learning strategies and activities.
Finally, we believe that students would greatly benefit from the integration of learning strategies in multiple courses during the first few semesters of their study program. One semester may not provide enough time and practice to change ingrained habits $[25,36,98]$.

\section{Strengths and limitations of this study}

A thematic analysis does not warrant claims of trends, correlations, or effects. Rather, its strength lies in providing a depth of understanding, providing descriptions of what some students' do and think. This provides us researchers with an improved understanding of potential processes in other samples before testing the effects. Whether our findings are likely to generalize to the students, treatments, settings, and outcomes relevant to the reader's context is a qualitative judgment that the reader must make [84].

The Covid-19 shutdown changed the intervention from a whole-semester intervention to a two-month intervention. The structured practice of the learning strategies was terminated after the shutdown, which forced the students to self-regulate their practice. However, the students' responses and ways of speaking indicated that their reported experiences and reflections were largely cemented before the shutdown. We found no indications in the interviews that our findings would have changed without the Covid-19 shutdown. One potential exception is retrieval practice where the students seemed to be gradually persuaded by the increasing benefits from additional practice. However, this was reported by the already-persuaded students, so the finding would most likely have been the same in a normal semester.

\section{Future research}

Further research needs to be done to find effective ways to integrate self-explanations and elaborative encoding into physics courses. One potential line of research is to develop instructional material for peer instruction of self-explanations and to analyze and optimize the learning processes. We also need to improve the task structure and instructional resources for elaborative encoding, ensuring that the associative links produced during elaborative encoding potentiates retrieval practice. More research should also be done to identify the intra- and inter-principle associative connections that are most useful for improving students' self-explanation and problem solving.

\section{ACKNOWLEDGMENTS}

This work was supported by the University of Bergen. We would like to thank Robert Gray Jr. for his advice on how to structure the interview guide. We would like to thank Matthias Gregor Stadler, Mildrid Kyte, and Vegard Havre Paulsen for valuable discussions of the thematic analysis. 
[1] M. Wells, D. Hestenes, and G. Swackhamer, A modeling method for high-school physics instruction, Am. J. Phys. 63, 606 (1995).

[2] D. Hestenes, Toward a modeling theory of physics instruction, Am. J. Phys. 55, 440 (1987).

[3] J. Larkin, J. McDermott, D. P. Simon, and H. A. Simon, Expert and novice performance in solving physics problems, Science 208, 1335 (1980).

[4] A. J. Mason and C. Singh, Surveying college introductory physics students' attitudes and approaches to problem solving, Eur. J. Phys. 37, 055704 (2016).

[5] M. T. H. Chi, P. J. Feltovich, and R. Glaser, Categorization and representation of physics problems by experts and novices, Cogn. Sci. 5, 121 (1981).

[6] B. Ibrahim, L. Ding, A. F. Heckler, D. R. White, and R. Badeau, Students' conceptual performance on synthesis physics problems with varying mathematical complexity, Phys. Rev. Phys. Educ. Res. 13, 010133 (2017).

[7] E. Mazur, Peer Instruction: A User's Manual, Prentice Hall Series in Educational Innovation (Prentice Hall, Upper Saddle River, NJ, 1997), p. 253.

[8] D. Hestenes, M. Wells, and G. Swackhamer, Force Concept Inventory, Phys. Teach. 30, 141 (1992).

[9] E. Kim and S.-J. Pak, Students do not overcome conceptual difficulties after solving 1000 traditional problems, Am. J. Phys. 70, 759 (2002).

[10] V. Gjerde, B. Holst, and S. D. Kolstø, Retrieval practice of a hierarchical principle structure in university introductory physics: Making stronger students, Phys. Rev. Phys. Educ. Res. 16, 013103 (2020).

[11] J. R. Anderson and C. D. Schunn, Implications of the ACTR Learning Theory: No Magic Bullets, in Advances in Instructional Psychology, Volume 5: Educational Design and Cognitive Science, edited by R. Glaser (Routledge, London, 2000), pp. 1-34.

[12] W. J. Hopper and D. E. Huber, Learning to recall: Examining recall latencies to test an intra-item learning theory of testing effects, J. Memory Lang. 102, 1 (2018).

[13] D. B. Markant, Active transitive inference: When learner control facilitates integrative encoding, Cognition $\mathbf{2 0 0}$, 104188 (2020).

[14] K. A. Ericsson and W. Kintsch, Long-term workingmemory, Psychol. Rev. 102, 211 (1995).

[15] P. T. Hardiman, R. Dufresne, and J. P. Mestre, The relation between problem categorization and problem-solving among experts and novices, Memory Cognit. 17, 627 (1989).

[16] M. T. H. Chi, M. Bassok, M. W. Lewis, P. Reimann, and R. Glaser, Self-explanations-How students study and use examples in learning to solve problems, Cogn. Sci. 13, 145 (1989).

[17] V. Gjerde et al.Problem solving in basic physics: Effective self-explanations based on four elements with support from retrieval practice (to be published).

[18] T. v. Gog, N. Rummel, and A. Renkl, Learning how to solve problems by studying examples, in The Cambridge Handbook of Cognition and Education (Cambridge University Press, New York, NY, 2019), pp. 183-208.

[19] P. D. Antonenko, C. A. Ogilvie, D. S. Niederhauser, J. Jackman, P. Kumsaikaew, R. R. Marathe, and S. M. Ryan,
Understanding student pathways in context-rich problems, Educ. Inf. Technol. 16, 323 (2011).

[20] A. Mason and C. Singh, Assessing expertise in introductory physics using categorization task. Phys. Rev. ST Phys. Educ. Res. 7, 020110 (2011).

[21] J. Dunlosky, K. A. Rawson, E. J. Marsh, M. J. Nathan, and D. T. Willingham, Improving students' learning with effective learning techniques: Promising directions from cognitive and educational psychology, Psychol. Sci. Publ. Interest 14, 4 (2013).

[22] H. L. Roediger and M. A. Pyc, Inexpensive techniques to improve education: Applying cognitive psychology to enhance educational practice, J. Appl. Res. Memory Cogn. 1, 242 (2012).

[23] T. Zu, J. Munsell, and N. S. Rebello, Comparing retrievalbased practice and peer instruction in physics learning, Phys. Rev. Phys. Educ. Res. 15, 010105 (2019).

[24] R. A. Bjork, J. Dunlosky, and N. Kornell, Self-regulated learning: Beliefs, techniques, and illusions, Annu. Rev. Psychol. 64, 417 (2013).

[25] J. Hattie, J. Biggs, and N. Purdie, Effects of learning skills interventions on student learning: A meta-analysis, Rev. Educ. Res. 66, 99 (1996).

[26] R. N. Blasiman, J. Dunlosky, and K. A. Rawson, The what, how much, and when of study strategies: comparing intended versus actual study behaviour. Memory 25, 784 (2017).

[27] J. M. Logan et al., Metacognition and the spacing effect: the role of repetition, feedback, and instruction on judgments of learning for massed and spaced rehearsal, Metacognition Learn. 7, 175 (2012).

[28] N. Kornell and L. K. Son, Learners' choices and beliefs about self-testing, Memory 17, 493 (2009).

[29] J. G. Tullis, J. R. Finley, and A. S. Benjamin, Metacognition of the testing effect: Guiding learners to predict the benefits of retrieval, Memory Cognit. 41, 429 (2013).

[30] V. X. Yan, E. L. Bjork, and R. A. Bjork, On the difficulty of mending metacognitive illusions: A priori theories, fluency effects, and misattributions of the interleaving benefit, J. Exp. Psychol. Gen. 145, 918 (2016).

[31] C. Dignath and M. V. J. Veenman, The role of direct strategy instruction and indirect activation of self-regulated learning-evidence from classroom observation studies, Educ. Psychol. Rev., https://doi.org/10.1007/s10648-02009534-0 (2020).

[32] A. Tricot and J. Sweller, Domain-specific knowledge and why teaching generic skills does not work, Educ. Psychol. Rev. 26, 265 (2014).

[33] O. O. Adesope, D. A. Trevisan, and N. Sundararajan, Rethinking the use of tests: A meta-analysis of practice testing (vol 87, pg 659, 2017), Rev. Educ. Res. 87, 659 (2017).

[34] S. C. Pan and T. C. Rickard, Transfer of test-enhanced learning: Meta-analytic review and synthesis, Psychol. Bull. 144, 710 (2018).

[35] R. A. R. Gurung and K. Burns, Putting evidence-based claims to the test: A multi-site classroom study of retrieval practice and spaced practice, Appl. Cogn. Psychol. 33, 732 (2019). 
[36] F. Biwer et al., Fostering effective learning strategies in higher education-A mixed-methods study, J. Appl. Res. Memory Cognit. 9, 186 (2020).

[37] T. Endres et al., Improving lifelong learning by fostering students' learning strategies at university, Psychol. Lear. Teach.-Plat., https://doi.org/10.1177/1475725720952025 (2020).

[38] D. L. Dinsmore, Strategic Processing in Education, Educ. Psych Insights (Routledge, New York, NY, 2018), p. 159.

[39] P. M. Gollwitzer, Implementation intentions-Strong effects of simple plans, Am. Psychol. 54, 493 (1999).

[40] R. E. Mayer, The Cambridge Handbook of Multimedia Learning, 2nd ed., Cambridge Handbooks in Psychology (Cambridge University Press, New York, 2014), p. 930.

[41] P. A. Alexander, The development of expertise: The journey from acclimation to proficiency, Educ. Res. 32, 10 (2003).

[42] See Supplemental Material at http://link.aps.org/ supplemental/10.1103/PhysRevPhysEducRes.17.010124 for contains the instructional tools, the original quotes with English translations, and the interview guide.

[43] C. A. Rowland, The effect of testing versus restudy on retention: A meta-analytic review of the testing effect, Psychol. Bull. 140, 1432 (2014).

[44] V. Braun and V. Clarke, Using thematic analysis in psychology, Qual. Res. Psychology 3, 77 (2006).

[45] J. A. Maxwell, The importance of qualitative research for causal explanation in education, Qual. Inquiry 18, 655 (2012).

[46] M. V. J. Veenman, B. H. A. M. Van Hout-Wolters, and P. Afflerbach, Metacognition and learning: Conceptual and methodological considerations. Metacogn. Learn. 1, 3 (2006).

[47] G. L. Bradshaw and J. R. Anderson, Elaborative encoding as an explanation of levels of processing, J. Verb. Learn. Verb. Behav. 21, 165 (1982).

[48] B.S. Stein et al., Elaboration and knowledge acquisition, Memory Cognit. 12, 522 (1984).

[49] J. Anderson and L. Reder, An elaborative processing explanation of depth processing, in Levels of Processing in Human Memory, edited by L. S. Cermak and F. I. M. Craik (Lawrence Erlbaum Associates; Hillsdale, N.Y., 1979).

[50] B.S. Stein and J. D. Bransford, Constraints on effective elaboration-Effects of precision and subject generation, J. Verb. Learn. Verb. Behav. 18, 769 (1979).

[51] J. R. Anderson, D. Bothell, M. D. Byrne, S. Douglass, C. Lebiere, and Y. Qin, An integrated theory of the mind, Psychol. Rev. 111, 1036 (2004).

[52] P. I. Pavlik and J. R. Anderson, Using a model to compute the optimal schedule of practice, J. Exp. Psychol. Appl. 14, 101 (2008).

[53] L. G. Eglington and P. I. Pavlik Jr., Optimizing practice scheduling requires quantitative tracking of individual item performance, Sci. Learn. 5, 15 (2020).

[54] M. Racsmány, Á. Szőllósi, and M. Marián, Reversing the testing effect by feedback is a matter of performance criterion at practice, Memory Cognit. 48, 1161 (2020).
[55] N. Kornell, R. A. Bjork, and M. A. Garcia, Why tests appear to prevent forgetting: A distribution-based bifurcation model, J. Memory Language 65, 85 (2011).

[56] W. J. Hopper and D. E. Huber, Testing the primary and convergent retrieval model of recall: Recall practice produces faster recall success but also faster recall failure, Memory Cognit. 47, 816 (2019).

[57] L. M. Reder, X. L. Liu, A. Keinath, and V. Popov, Building knowledge requires bricks, not sand: The critical role of familiar constituents in learning. Psychon. Bull. Rev. 23, 271 (2016).

[58] Z. F. Shen et al., Item strength affects working memory capacity, Memory Cognit. 46, 204 (2018).

[59] M. C. Lovett, L. Z. Daily, and L. M. Reder, A source activation theory of working memory: cross-task prediction of performance in ACT-R, Cogn. Systems Res. 1, 99 (2000).

[60] J. R. Anderson, J. M. Fincham, and S. Douglass, Practice and retention: A unifying analysis, J. Exper. Psychol.Learn. Memory Cognit. 25, 1120 (1999).

[61] A. C. Butler, Repeated testing produces superior transfer of learning relative to repeated studying, J. Exper. Psychol.Learn. Memory Cognit. 36, 1118 (2010).

[62] S. K. Carpenter, Testing enhances the transfer of learning, Curr. Dir. Psychol. Sci. 21, 279 (2012).

[63] D. Rohrer, K. Taylor, and B. Sholar, Tests enhance the transfer of learning, J. Exper. Psychol.-Learn. Memory Cognit. 36, 233 (2010).

[64] B. Pastotter and K. H. Bauml, Retrieval practice enhances new learning: The forward effect of testing, Front. Psychol. 5, 286 (2014).

[65] J. C. K. Chan, C. A. Meissner, and S. D. Davis, Retrieval potentiates new learning: A theoretical and meta-analytic review, Psychol. Bull. 144, 1111 (2018).

[66] K. T. Wissman, K. A. Rawson, and M. A. Pyc, The interim test effect: Testing prior material can facilitate the learning of new material, Psychon. Bull. Rev. 18, 1140 (2011).

[67] J. R. Anderson and J. M. Fincham, Extending problemsolving procedures through reflection, Cogn. Psychol. 74, 1 (2014).

[68] J. J. Williams and T. Lombrozo, The role of explanation in discovery and generalization: Evidence from category learning, Cogn. Sci. 34, 776 (2010).

[69] J. J. Williams and T. Lombrozo, Explanation and prior knowledge interact to guide learning, Cogn. Psychol. 66, 55 (2013).

[70] R. M. F. Wong, M. J. Lawson, and J. Keeves, The effects of self-explanation training on students' problem solving in high-school mathematics, Learn. Instr. 12, 233 (2002).

[71] A. Renkl, Learning from worked-out examples: A study on individual differences, Cogn. Sci. 21, 1 (1997).

[72] J. R. Anderson, J. M. Fincham, and S. Douglass, The role of examples and rules in the acquisition of a cognitive skill, J. Exper. Psychol.-Learn. Memory Cognit. 23, 932 (1997).

[73] V. Aleven and K. R. Koedinger, An effective metacognitive strategy: Learning by doing and explaining with a computer-based cognitive tutor, Cogn. Sci. 26, 147 (2002).

[74] H. S. Lee, S. Betts, and J. R. Anderson, Embellishing problem-solving examples with deep structure information facilitates transfer, J. Exp. Educ. 85, 309 (2017). 
[75] L. Deslauriers, L. S. McCarty, K. Miller, K. Callaghan, and G. Kestin, Measuring actual learning versus feeling of learning in response to being actively engaged in the classroom, Proc. Natl. Acad. Sci. U.S.A. 116, 19251 (2019).

[76] A. Kirk-Johnson, B. M. Galla, and S. H. Fraundorf, Perceiving effort as poor learning: The misinterpreted-effort hypothesis of how experienced effort and perceived learning relate to study strategy choice, Cogn. Psychol. 115, 101237 (2019).

[77] H. D. Young, R. A. Freedman, and H. D. Young, University Physics with Modern Physics, 13th ed. (Pearson Higher Education, Hoboken, NJ, 2012).

[78] H.S. Lee et al., An fMRI investigation of instructional guidance in mathematical problem solving, Trends Neurosci. Educ. 3, 50 (2014).

[79] J. E. Richey and T. J. Nokes-Malach, How much is too much? Learning and motivation effects of adding instructional explanations to worked examples, Learn. Instr. 25, 104 (2013).

[80] QSR International, NVivo 12 Pro. 2018, QSR International: United States.

[81] D. An and M. Carr, Learning styles theory fails to explain learning and achievement: Recommendations for alternative approaches, Personality Indiv. Diff. 116, 410 (2017).

[82] W. A. Firestone, Alternative arguments for generalizing from data as applied to qualitative research, Educ. Res. 22, 16 (1993).

[83] L. J. Cronbach, Beyond the two disciplines of scientific psychology, Am. Psychol. 30, 116 (1975).

[84] Y.S. Lincoln and E. G. Guba, Naturalistic Inquiry (Sage Publications, Beverly Hills, CA, 1985), p. 416.

[85] R. K. Yin, Case Study Research: Design and Methods (Sage Publications, London, 1989).

[86] A. Robey, The benefits of testing: Individual differences based on student factors, J. Mem. Lang. 108, 104029 (2019).

[87] F. M. van Blankenstein, D. H. J. M. Dolmans, C. P. M. van der Vleuten, and H. G. Schmidt, Which cognitive processes support learning during small-group discussion? The role of providing explanations and listening to other, Instr. Sci. 39, 189 (2011).
[88] J. D. Karpicke and M. A. Smith, Separate mnemonic effects of retrieval practice and elaborative encoding, J. Memory Lang. 67, 17 (2012).

[89] M. Abel and K.H. T. Bauml, Would you like to learn more? Retrieval practice plus feedback can increase motivation to keep on studying, Cognition 201, 104316 (2020).

[90] T. J. Nokes-Malach, K. VanLehn, D. M. Belenky, M. Lichtenstein, and G. Cox, Coordinating principles and examples through analogy and self-explanation, Eur. J. Psychol. Educ. 28, 1237 (2013).

[91] C. D. Morris, J. D. Bransford, and J. J. Franks, Levels of processing versus transfer appropriate processing, J. Verb. Learn. Verb. Behav. 16, 519 (1977).

[92] A. Renkl, Toward an instructionally oriented theory of example-based learning, Cogn. Sci. 38, 1 (2014).

[93] A. Renkl and R. K. Atkinson, Structuring the transition from example study to problem solving in cognitive skill acquisition: A cognitive load perspective, Educ. Psychol. 38, 15 (2003).

[94] M. Good, E. Marshman, E. Yerushalmi, and C. Singh, Graduate teaching assistants' views of broken-into-parts physics problems: Preference for guidance overshadows development of self-reliance in problem solving, Phys. Rev. Phys. Educ. Res. 16, 010128 (2020).

[95] R. Badeau, D. R. White, B. Ibrahim, L. Ding, and A. F. Heckler, What works with worked examples: Extending self-explanation and analogical comparison to synthesis problems. Phys. Rev. Phys. Educ. Res. 13, 020112 (2017).

[96] E. Marshman, S. DeVore, and C. Singh, Holistic framework to help students learn effectively from researchvalidated self-paced learning tools, Phys. Rev. Phys. Educ. Res. 16, 020108 (2020).

[97] A. Renkl et al., Training principle-based self-explanations: Transfer to new learning contents, in Proceedings of the 35th Annual Conference of the Cognitive Science Society (2013), pp. 1205-1210.

[98] A. S. Donker, H. de Boer, D. Kostons, C. C. Dignath van Ewijk, and M. P. C. van der Werf, Effectiveness of learning strategy instruction on academic performance: A metaanalysis. Educ. Res. Rev. 11, 1 (2014). 